\title{
Susceptibilidade do besouro rola-bosta africano a reguladores de crescimento de insetos
}

\author{
[Susceptibility of African dung beetle to insect growth regulators] \\ L.N. Domingues, J. Mendes* \\ Instituto de Ciências Biomédicas - UFU \\ Caixa Postal 593 \\ 38405-302 - Uberlândia, MG
}

\begin{abstract}
RESUMO
Verificou-se a ação dos reguladores de crescimento de insetos (IGR), diflubenzuron e methoprene, sobre o besouro rola-bosta africano, Digitonthophagus gazella (Fabricius), um inimigo natural da mosca-doschifres, Haematobia irritans irritans (Linnaeus). Casais de besouros foram colocados em baldes contendo terra úmida e alimentados com fezes bovinas contendo 1, 0,5 ou 0,2ppm de diflubenzuron e $0,2 \mathrm{ppm}$ de methoprene. Os insetos e sua prole foram recuperados com o auxílio de armadilhas pitfall. Diflubenzuron e methoprene não afetaram a sobrevivência dos adultos inicialmente expostos, mas interferiram na produção de descendentes. Diflubenzuron, nas concentrações de 1 e $0,5 \mathrm{ppm}$, também afetou a duração do ciclo de vida dos besouros. Nenhum dos IGRs alterou a razão sexual dos descendentes obtidos. As concentrações testadas de diflubenzuron mostraram-se moderadamente nocivas ao besouro enquanto methoprene a $0,2 \mathrm{ppm}$ mostrou ser pouco nocivo, segundo os critérios da International Organization for Biological Control.
\end{abstract}

Palavras-chave: besouro rola-bosta, Digitonthophagus gazella, diflubenzuron, methoprene, inimigo natural

\section{ABSTRACT}

The effects of insect growth regulators (IGR), diflubenzuron and methoprene, on African dung beetle, Digitonthophagus gazella (Fabricius), a natural enemy of the horn fly, Haematobia irritans irritans (Linnaeus), was studied. Beetles were placed in buckets partially filled with humid soil and were fed bovine feces containing 1, 0.5, or 0.2ppm diflubenzuron and 0.2ppm methoprene. Insects and their progenies were recovered by pitfall traps. Diflubenzuron and methoprene did not affect the survival of the adults but reduced their progenies. Diflubenzuron 1 and 0.5 ppm also affected the life cicle of the beetles. None of the IGR modified the gender ratio of the progenies. According to the IOBC criteria, diflubenzuron tested concentrations showed to be moderately harmful to the beetles, whereas methoprene $0.2 p p m$ was slightly harmful.

Keywords: African dung beetle, Digitonthophagus gazella, methoprene, diflubenzuron, natural enemy

\section{INTRODUÇÃO}

Os reguladores de crescimento de insetos (IGR) atuam durante os processos de metamorfose e muda dos insetos, impedindo o desenvolvimento dos imaturos até o estádio adulto. São considerados mais seletivos e específicos que os inseticidas convencionais por agirem diretamente no sistema hormonal dos insetos (Chamberlain, 1975; Graff, 1993). Diflubenzuron e methoprene são IGR pertencentes à classe dos inibidores da síntese de quitina e análogos ao hormônio juvenil dos insetos, respectivamente (Graff, 1993).

Recebido em 1 de fevereiro de 2008

Aceito em 24 de agosto de 2009

E-mail: jmendes@ufu.br 
O besouro rola-bosta africano, Digitonthophagus gazella (Fabricius, 1787) (Coleoptera: Scarabaeidae), foi introduzido no Brasil pelo Centro Nacional de Pesquisa de Gado de Corte da Empresa Brasileira de Pesquisa Agropecuária como uma das estratégias do Programa Integrado de Controle da Mosca-dos-chifres (Nascimento et al., 1990). Este besouro é inimigo natural da mosca-dos-chifres, Haematobia irritans irritans (Linnaeus, 1758) (Diptera: Muscidae), pois enterra e desestrutura as fezes bovinas, destruindo o habitat de suas larvas (Blume et al., 1973).

A eficiência de diflubenzuron e de methoprene contra H. irritans é bem conhecida (Gingrich e Hopkins, 1976; Kunz et al., 1976; Kunz e Bay, 1977; Fincher, 1991; Scott et al., 1995; Silva e Mendes, 2002). Entretanto, há poucas informações sobre a ação desses IGR na artropodofauna não alvo presente nas fezes bovinas, grande parte considerada benéfica (Wardhaug, 2005). Os escarabeídeos são importantes na degradação das fezes bovinas, auxiliando a ciclagem dos nutrientes e a higienização das pastagens (Miranda et al., 2000), além de auxiliarem no controle da moscados-chifres (Blume et al., 1973). Assim, estudos sobre efeitos de drogas antiparasitárias sobre organismos não alvos que se alimentam e/ou se desenvolvem nos excrementos dos animais são de relevância ecológica e econômica.

Os objetivos deste trabalho foram: verificar a susceptibilidade de $D$. gazella ao diflubenzuron e methoprene, a interferência desses IGR na duração do ciclo de vida dos insetos, e na produção e razão sexual dos seus descendentes. A toxicidade dos IGR também foi analisada, utilizando critérios de seletividade da IOBC (Working..., 2005).

\section{MATERIAL E MÉTODOS}

Os experimentos foram realizados entre os meses de abril de 2005 e dezembro de 2006. Todos os besouros utilizados nos experimentos originaram-se da colônia da Empresa de Pesquisa Agropecuária de Minas Gerais, situada em Uberaba, MG.

Solução estoque de diflubenzuron foi preparada com base na dissolução de $100 \mathrm{mg}$ de diflubenzuron a $25 \%$ de ingrediente ativo (IA) ${ }^{1}$ em $250 \mathrm{~mL}$ de acetona PA. Alíquotas de 18,9 e $3,6 \mathrm{~mL}$ dessa solução foram adicionadas e misturadas mecanicamente por aproximadamente 15 minutos a $1,8 \mathrm{~kg}$ de fezes bovinas recém defecadas, colhidas de animais que não haviam recebido nenhum tratamento para o controle de ecto- ou endoparasitos. Tal procedimento resultou na produção de meios de criação com uma parte por milhão (1ppm), 0,5ppm e 0,2ppm de diflubenzuron. Solução estoque de methoprene foi preparada de maneira semelhante, pela dissolução de $40 \mathrm{mg}$ de Methoprene a $1,5 \% \mathrm{IA}^{2}$ em $120 \mathrm{~mL}$ de acetona PA. Em seguida, $40 \mathrm{ml}$ dessa solução foram adicionados a $1,0 \mathrm{~kg}$ de fezes, resultando em um meio com $0,2 \mathrm{ppm}$ de methoprene. Todos os meios ficavam em repouso à temperatura ambiente durante 12 horas antes de serem oferecidos aos besouros.

Nos experimentos em que os besouros adultos foram expostos ao diflubenzuron nas concentrações de 1 e $0,2 \mathrm{ppm}$ e ao methoprene na concentração de $0,2 \mathrm{ppm}$, utilizaram-se 10 baldes com quatro casais de besouros cada. Nos experimentos com diflubenzuron a $0,5 \mathrm{ppm}$, foram utilizados 12 baldes para o grupo tratado e seu respectivo grupo-controle, ambos com quatro casais de besouros. No segundo experimento realizado com methoprene, também na concentração de 0,2ppm, utilizaram-se oito baldes para o grupo-controle e seis baldes para os grupos tratados, pois o número de besouros a ser exposto dependeu da prole oriunda dos coleópteros tratados durante todo seu ciclo de vida. Cada balde coberto com organza recebeu os besouros e $200 \mathrm{~g}$ dos respectivos substratos de alimentação. As fezes oferecidas aos besouros dos grupos tratados continham a concentração do IGR a ser testada, enquanto as fezes dos gruposcontrole continham somente acetona.

Durante 10 dias consecutivos, fezes foram colocadas à vontade nos baldes, e a umidade da terra foi sempre restabelecida. No $11^{\circ}$ dia, retiraram-se as fezes remanescentes e armadilhas do tipo pitfall foram enterradas até suas bordas, para captura dos adultos (F0). Essa captura ocorreu durante cinco dias consecutivos. Após

${ }^{1}$ Difly ${ }^{\circledR}$, Champion Farmoquímico Ltda. - Anápolis, Brasil.

${ }^{2}$ Altosid XR-G ${ }^{\mathbb{Q}}$; Novartis Saúde Animal Ltda. - São Paulo, Brasil. 
estes dias, as fezes, presentes nas armadilhas, foram retiradas e não repostas por um período de 15 dias. A partir de então, fezes foram novamente colocadas nas armadilhas e monitoradas, diariamente, com o objetivo de coletar a prole adulta (F1), proveniente dos besouros introduzidos inicialmente nos baldes. Passados 15 dias de coletas diárias da prole, a terra de cada balde foi peneirada com o intuito de encontrar besouros ou peras contendo larvas ou pupas remanescentes.

Foi realizado um experimento adicional com methoprene seguindo a mesma metodologia anteriormente descrita. Parte da prole (F1), oriunda dos primeiros adultos expostos (F0), não foi exposta ao IGR (grupo tratado I) e outra parte foi novamente exposta ao IGR na concentração de $0,2 \mathrm{ppm}$ (grupo tratado II). O grupo-controle constituiu-se de besouros que não receberam tratamento durante todo o seu desenvolvimento. Desse experimento obteve-se uma segunda geração de besouros (F2).

Durante todo o período de realização dos experimentos, um aquecedor foi ligado no local de criação dos besouros, visando evitar grandes oscilações de temperatura, nos dias e noites mais frios. Também, foi colocado no local um termoigrômetro para monitoramento diário da temperatura e da umidade.

Os resultados obtidos foram inicialmente analisados quanto ao ajuste à distribuição normal pelo teste Kolmogorov-Smirnov. Em seguida, foram comparados utilizando os testes KruskalWallis ou análise de variância. Nos casos em que mais de dois grupos foram comparados pelas análises de variância e mostraram-se diferentes, o teste Tukey foi aplicado posteriormente. Adotou-se o nível de significância de 0,05 para todas as análises. Os testes foram feitos com o auxílio do software Systat (Systat..., 2005).

Os dados também foram analisados pelos critérios da escala de seletividade da IOBC (Working..., 2005). Segundo essa escala, o inseticida que causa redução de menos de $30 \%$ na produção de descendentes por insetos adultos que tenham sido expostos a ele em laboratório é considerado um produto inócuo; aquele que causa redução de 30 a $79 \%$ é considerado um produto pouco nocivo; aquele que causa redução de $80 \%$ a $99 \%$ é considerado um produto moderadamente nocivo, e o que causa mais de $99 \%$ de redução na produção de descendentes é considerado um produto nocivo ao inseto.

\section{RESULTADOS E DISCUSSÃO}

A média das temperaturas do período em que foi realizado o presente estudo $\left(27,5^{\circ} \mathrm{C} \pm 2,37\right)$ está próxima da temperatura de $28^{\circ} \mathrm{C}$ preconizada para criação de D. gazella em laboratório (Nascimento et al., 1990; Martins e Contel, 1997).

Nenhuma das concentrações testadas de diflubenzuron e methoprene teve influência sobre a sobrevivência dos adultos de D. gazella, mas alteraram significativamente a produção de seus descendentes (Tab. 1 e 2). As alterações podem ter ocorrido tanto por atuação negativa dos IGR no desenvolvimento dos imaturos até a fase adulta, quanto pela redução na fecundidade/fertilidade desses adultos.

Tabela 1. Sobrevivência de adultos e produção de descendentes (F1) de Digitonthophagus gazella expostos ao inibidor de crescimento de insetos diflubenzuron (1, 0,5 e 0,2ppm)

\begin{tabular}{lccccr} 
Grupo & $\begin{array}{c}\mathrm{N}^{\mathrm{o}} \text { inicial de } \\
\text { besouros }\end{array}$ & $\begin{array}{c}\text { Adultos } \\
\text { recuperados }\end{array}$ & $\begin{array}{c}\text { Prole } \\
\text { obtida }\end{array}$ & Razão sexual & $\begin{array}{c}\text { Proporção de } \\
\text { prole obtida }^{(1)}\end{array}$ \\
\hline Controle & 80 & $50 \mathrm{~A}$ & $321 \mathrm{a}$ & $0,63 \mathrm{~A}$ & 1 \\
$1 \mathrm{ppm}$ & 80 & $50 \mathrm{~A}$ & $14 \mathrm{~b}$ & $0,79 \mathrm{~A}$ & 0,04 \\
Controle & 96 & $58 \mathrm{~A}$ & $130 \mathrm{a}$ & $0,48 \mathrm{~A}$ & 1 \\
$0,5 \mathrm{ppm}$ & 96 & $62 \mathrm{~A}$ & $8 \mathrm{~b}$ & $0,50 \mathrm{~A}$ & 0,06 \\
Controle & 80 & $35 \mathrm{~A}$ & $60 \mathrm{a}$ & $0,42 \mathrm{~A}$ & 1 \\
$0,2 \mathrm{ppm}$ & 80 & $37 \mathrm{~A}$ & $15 \mathrm{~b}$ & $0,73 \mathrm{~A}$ & 0,25 \\
\hline
\end{tabular}

(1) Proporção de prole obtida em relação ao grupo-controle. Valores seguidos por letras distintas maiúsculas ou minúsculas na coluna diferem entre si $(\mathrm{P}<0,05)$ pelo teste Kruskall-Wallis e pelo, a posteriori, teste Tukey, respectivamente. 
Tabela 2. Sobrevivência de adultos e produção de descendentes (F1) de Digitonthophagus gazella expostos ao methoprene $(0,2 \mathrm{ppm})$

\begin{tabular}{lccccc}
\hline Grupo & $\begin{array}{c}\mathrm{N}^{\circ} \text { inicial de } \\
\text { besouros }\end{array}$ & $\begin{array}{c}\text { Adultos } \\
\text { recuperados }\end{array}$ & Prole obtida & Razão sexual & $\begin{array}{c}\text { Proporção } \\
\text { de prole } \\
\text { obtida }{ }^{(1)}\end{array}$ \\
\hline Controle & 80 & $50 \mathrm{~A}$ & $321 \mathrm{a}$ & $0,63 \mathrm{~A}$ & 1 \\
$0,2 \mathrm{ppm}$ & 80 & $44 \mathrm{~A}$ & $129 \mathrm{~b}$ & $0,59 \mathrm{~A}$ & 0,39 \\
\hline
\end{tabular}

${ }^{(1)}$ Proporção de prole obtida em relação ao grupo-controle. Valores seguidos por letras distintas maiúsculas ou minúsculas na coluna diferem entre si $(\mathrm{P}<0,05)$ pelo teste Kruskall-Wallis e pelo teste F, respectivamente.

Diflubenzuron age durante os processos de muda e desenvolvimento dos imaturos, interferindo na síntese e/ou deposição de quitina durante esse momento. Assim, as larvas não conseguem deixar a cutícula precedente, não completam a ecdise e morrem antes de chegarem ao estádio de pupa (Tunaz e Uygun, 2004). Methoprene mimetiza fielmente a ação do hormônio juvenil e atua sobre o desenvolvimento embrionário dos ovos e/ou retarda o desenvolvimento das pupas, acarretando mortalidade dos imaturos (Wilson, 2004). Também não se pode descartar a hipótese de um efeito direto do methoprene sobre os adultos, interferindo no comportamento reprodutivo, na produção de feromônios e na oogênese dos insetos (Moczeck e Nijhout, 2002).

A interferência de diflubenzuron na produção de descendentes por adultos de D. gazella e de outros insetos não alvos já foi relatada por outros autores (Pickens e Miller, 1975; Fincher, 1991). Ciociola Junior (2006) ofereceu a bovinos sal mineral com diflubenzuron na proporção de $15 \mathrm{~g} / 30 \mathrm{~kg}$ e posteriormente utilizou as fezes dos animais na manutenção de D. gazella. $\mathrm{O}$ autor observou redução de $21 \%$ no número de descendentes produzidos pelo besouro. Segundo os critérios da IOBC (Working..., 2005), a concentração do produto presente nas fezes utilizadas por Ciociola Junior (2006) seria considerada inócua, enquanto os resultados encontrados no presente estudo indicariam que diflubenzuron a 1 e $0,5 \mathrm{ppm}$ é moderadamente nocivo e a $0,2 \mathrm{ppm}$ é pouco nocivo para $D$. gazella (Tab. 1). A menor redução na produção de descendentes, observada por Ciociola Junior (2006), parece dever-se à exposição dos besouros a concentrações do IGR menores que as testadas neste estudo. As concentrações aqui testadas são também maiores que as verificadas por Silva e Mendes (2002) como eficientes para o controle da mosca-dos-chifres. A aplicação de técnicas de mensuração das concentrações de diflubenzuron presentes nas fezes de bovinos tratados, conforme prescrições dos fabricantes do produto comercial, pode confirmar a hipótese acima e indicar concentrações adicionais de diflubenzuron, inclusive mais baixas que as aqui utilizadas, para serem testadas em espécies não alvos.

Methoprene causou redução de $70 \%$ na produção de proles nos experimentos (Tab. 2 e 3), o que, segundo os critérios da IOBC, o leva a ser classificado como um inseticida pouco nocivo a D. gazella. Blume et al. (1974) relataram que methoprene nas concentrações de 100, 10, 5 e $1 \mathrm{ppm}$, diretamente misturado às fezes bovinas, inibiu a eclosão de ovos de $D$. gazella. Entretanto, Fincher (1991) não notou interferência desse IGR na produção de descendentes deste coleóptero. Lastro (2006) também não relatou interferência desse inseticida sobre Onthophagus taurus, outro controlador natural de $H$. irritans. Methoprene a 0,2ppm é considerado efetivo no controle da mosca-doschifres (Gingrich e Hopkins, 1976). Também há registro da efetividade deste IGR sobre a mosca a 0,08ppm (Lastro, 2006), concentração ainda menor que a testada no presente estudo. As considerações feitas acima para diflubenzuron, quanto a futuros testes indicarem outras concentrações a serem testadas para aquele IGR, também são válidas para methoprene.

Methoprene não afetou a sobrevivência dos adultos do besouro, independentemente de eles, quando eram imaturos, terem sido expostos ou não ao IGR. O IGR também não interferiu significativamente na produção de uma segunda geração de descendentes (Tab. 3). No entanto, deve-se considerar que, em razão do baixo 
número de descendentes obtidos em todos os grupos no experimento em que se obteve a segunda geração de besouros em laboratório, os resultados obtidos não devem ser considerados conclusivos. Deve-se ressaltar que Blume et al. (1974), quando transferiram adultos de $D$. gazella que haviam sido tratados com methoprene a 5 e $1 \mathrm{ppm}$ para meios que não continham o IGR, verificaram que os besouros não sofreram nenhuma alteração em sua fecundidade.

Tabela 3. Sobrevivência de adultos (F1) e produção de descendentes (F2) de Digitonthophagus gazella expostos ao Methoprene (0,2 ppm)

\begin{tabular}{lccccc}
\hline Grupo & $\begin{array}{c}\mathrm{N}^{\mathrm{o}} \text { inicial de } \\
\text { besouros }\end{array}$ & $\begin{array}{c}\text { Adultos } \\
\text { recuperados }\end{array}$ & Prole obtida & $\begin{array}{c}\text { Razão sexual } \\
\begin{array}{c}\text { Proporção de } \\
\text { prole obtida } \\
(1)\end{array}\end{array}$ \\
\hline Controle & 64 & 29 & 34 & 0,65 & 1 \\
Tratado 1 & 48 & 26 & 8 & 0,62 & 0,31 \\
Tratado 2 & 48 & 21 & 10 & 0,80 & 0,39 \\
\hline
\end{tabular}

${ }^{(1)}$ Proporção de prole obtida em relação ao grupo-controle. Não houve diferença entre os grupos $(\mathrm{P}<0,05)$.

Blume e Aga (1975) e Martins e Contel (1997), ao criarem D. gazella em laboratório, verificaram, respectivamente, médias de 29,8 e 38,5 dias de desenvolvimento de ovo até adulto. A última média está bem próxima das encontradas em todos os grupos de besouros do presente estudo. No entanto, houve diferença significativa entre a duração do ciclo de vida do grupo-controle e os grupos tratados com diflubenzuron a 1 e $0,5 \mathrm{ppm}$ (Fig. 1). Não há registros prévios na literatura consultada de alteração na duração do ciclo de vida de $D$. gazella por diflubenzuron.

Methoprene a 0,2ppm não afetou a duração do ciclo de vida da primeira geração de descendentes $(\mathrm{H}=0,446 ; 1 ; \mathrm{P}=0,504)$, mas alterou a duração do ciclo de vida da segunda geração de besouros a ele exposta (Fig. 2). Mais uma vez, aqui seria prudente considerar o baixo número de descendentes obtidos em todos os grupos do experimento, não possibilitando a obtenção de resultados conclusivos. Ressalte-se que Lastro (2006) não verificou interferência de methoprene na duração do ciclo de vida da prole F1 de $O$. taurus.

Vários autores já observaram maior proporção de fêmeas nas proles de D. gazella (Blume e Aga,
1975; Martins e Contel, 1997; Ciociola Júnior, 2006). No presente estudo, também foram observadas progênies com maior proporção de fêmeas na maioria dos grupos e não houve diferenças nas razões sexuais entre os descendentes de todos os grupos tratados e não tratados (Tab. 1, 2, 3), demonstrando que os IGRs não interferiram na razão sexual desse besouro. Não há registros na literatura consultada de interferência de methoprene na razão sexual de coleópteros. Roth (1989) constatou a interferência desse IGR na razão sexual de Spalangia cameroni (Perkins, 1910) (Hymenoptera: Pteromalidae), um parasitoide da mosca-dos-chifres.

Diante dos resultados apresentados, conclui-se que: as concentrações testadas de diflubenzuron e methoprene não interferem na sobrevivência de D. gazella, quando exposto na forma adulta e nem na razão sexual da sua prole, mas a produção de descendentes é reduzida significativamente. Logo, as concentrações testadas de diflubenzuron e methoprene mostram-se moderadamente nocivas e pouco nocivas ao besouro, respectivamente. 


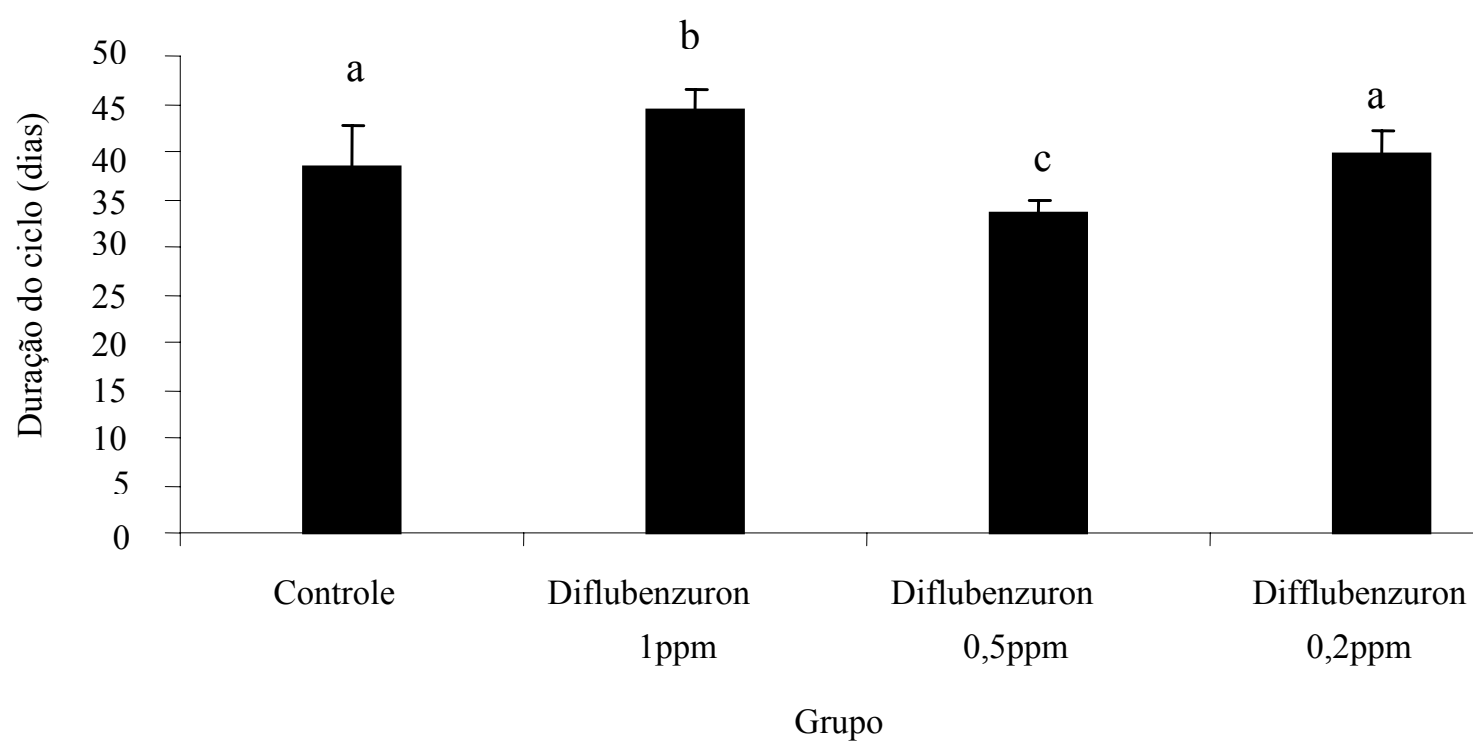

Figura 1. Duração do ciclo de vida de besouros expostos ao diflubenzuron (1, 0,5 e 0,2ppm) desde o acasalamento até a emergência de adultos (F1). Valores seguidos por letras distintas diferem entre si $(\mathrm{P}<0,05)$ pelo, a posteriori, teste Tukey. Barras acima das colunas referem-se aos erros-padrão; ppm $=$ partes por milhão

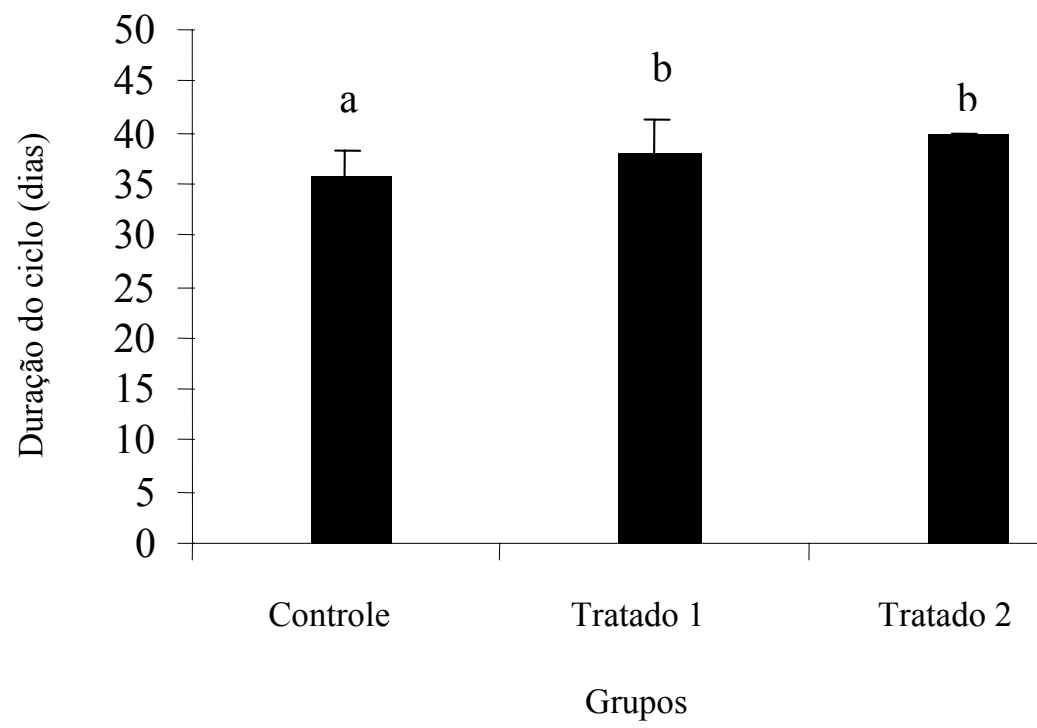

Figura 2. Duração do ciclo de vida de besouros expostos ao methoprene (0,2ppm) desde o acasalamento (F1) até a emergência de adultos (F2). Valores seguidos por letras distintas diferem entre si $(\mathrm{P}<0,05)$ pelo, a posteriori, teste Tukey. Barras acima das colunas referem-se aos erros-padrão; ppm = partes por milhão. 


\section{AGRADECIMENTOS}

Ao Dr. Américo Iorio Ciociola Júnior, EPAMIG de Uberaba, por nos disponibilizar o laboratório para a realização de um dos experimentos. À Capes, pelo auxílio financeiro e às empresas Champion Farmoquímico e Novartis Saúde Animal, por terem fornecido gratuitamente os produtos testados.

\section{REFERÊNCIAS BIBLIOGRÁFICAS}

BLUME, R.R.; AGA, A. Onthophagus gazella: mass rearing and laboratory biology. Environ. Entomol.,v.4, p.735-736, 1975.

BLUME, R.R.; AGA, A.; OEHLER, D.D. et al. Onthophagus gazella: a non-target arthropod for the evaluation of bovine feces containing Methoprene. Environ. Entomol., v.3, p.947-949, 1974.

BLUME, R.R.; MATTER, J.J; ESCHLE, J.L. Onthophagus gazella: effect on survival of horn flies in the laboratory. Environ. Entomol., v.2, p.811-813, 1973.

CHAMBERLAIN, W.F. Insect growth regulating agents for control of arthropods of medical and veterinary importance. J. Med. Entomol., v.12, p.395-400, 1975.

CIOCIOLA JUNIOR, A.I.; Efeito do produto Difly no desenvolvimento do besouro africano, Digitonthophagus gazella (Coleoptera: Scarabaeidae) em laboratório. In: CONGRESSO BRASILEIRO DE MEDICINA VETERINARIA, 33., 2006, Cuiabá. Anais... Cuiabá: Conbravet, 2006. p.161-162.

FINCHER, G.T. Sustained-release bolus for horn fly (Diptera: Muscidae) control: effects of Methoprene and Diflubenzuron on some nontarget species. Environ. Entomol., v.20, p.7782, 1991.

GINGRICH, A.R.; HOPKINS, D.E. Stages of the horn fly susceptible to Methoprene. J. Econ. Entomol., v.70, p.107-108, 1976.

GRAFF, J.F. The role of insect growth regulators in arthropod control. Parasitol. Today, v.9, p.471-474, 1993.

KUNZ, S.E.; BAY, D.E. Diflubenzuron: Effects on the fecundity, production and longevity of the horn fly. Southwest Entomol., v.2, p.27-31, 1977.
KUNZ, S.E.; SCHIMDT, C.D.; HARRIS, R.L. Effectiveness of Diflubenzuron applied as dust to inhibit reproduction in horn flies. Southwest Entomol., v.1, p.190-193, 1976.

LASTRO, E. Dung beetles (Coleoptera: Scarabaeidae and Geotrupidae) in North Carolina pastures ecosystem. 2006. 134f. Thesis (Master of Science) - Entomology Department, Graduate Faculty of North Carolina State University, North Carolina.

MARTINS, E.; CONTEL, E.P.B. Dados biológicos da criação do besouro africano Onthophagus gazella Fabricius (Scarabaeidae) em terrários na Fazenda Experimental Getúlio Vargas de Uberaba (MG). Rev. Bras. Biol., v.57, p.403-409, 1997.

MIRANDA, C.H.B.; SANTOS, J.C.C.; BIANCHIN, I. The role of Digitonthophagus gazella in pasture cleaning and production as a result of burial of cattle dung. Pasturas Trop., v.22, p.14-18, 2000.

MOCZECK, A.P.; NIJHOUT, H.F. Developmental mechanisms of threshold evolution in a polyphonic beetle. Evol. Develop., v.4, p.252-264, 2002

NASCIMENTO, Y.A.; BIANCHIN, I.; HONER, M.R. Instruções para a criação do besouro africano Onthophagus gazella em laboratório. Campo Grande: Embrapa, 1990. p.1-5. (Comunicado Técnico, n. 33).

PICKENS, L.G.; MILLER. R.W. Growth regulating chemicals tested against nontarget insect fauna in bovine fecal pats. Environ. Entomol., v.4, p.46-48, 1975.

ROTH, J.P. Some effects of Methoprene on Spalangia cameroni, a parasitoid of horn fly pupae. Southwest Entomol., v.14, p.91-96, 1989.

SCOTT, F.B.; GRISI, L.; COMENDOUROS, K. Avaliação a nível de campo do uso de Methoprene, adicionado ao sal mineral, no controle de Haematobia irritans em bovinos. Rev. Bras. Parasitol. Vet., v.4, p.85-88, 1995.

SILVA, J.J.; MENDES, J. Effect of diflubenzuron on imature stages of Haematobia irritans (L.) (Diptera: Muscidae) in Uberlândia, State of Minas Gerais, Brazil. Mem. Inst. Oswaldo Cruz, v.97, p.679-682, 2002.

SYSTAT $^{\circledR}$ for Windows ${ }^{\circledR}$. 2005. Version 11 [S.I.]: Systat Software, 1 CD-ROM. 
TUNAZ, H.; UYGUN, N. Insect growth regulators for insect pest control. Turk. J. Agric. For., v.28, p.377-387, 2004.

WARDHAUGH, K.G. Insecticidal activity of synthetic pyrethroids, organophosphates, insect growth regulators and other livestock parasiticides: an australian perspective. Environ. Toxicol. Chem., v.24, p.789-796, 2005.

WILSON, T.G. The molecular site of action of juvenile hormone and juvenile hormone insecticides during metamorphosis: how these compounds kill insects. J. Insect. Physiol., v.50, p.11-121, 2004.

WORKING document on selectivity of pesticides. International Organization for Biological Control, 2005. Disponível em: $<$ www.iobc.ch/2005/Working\%20Document $\% 20$ Pesticides_Explanations.pdf $>$ Acessado em: 14 jan. 2006. 